Canad. Math. Bull. Vol. 26 (4), 1983

\title{
THE HEIGHT OF TWO-DIMENSIONAL COHOMOLOGY CLASSES OF COMPLEX FLAG MANIFOLDS
}

BY

\author{
S. ALLEN BROUGHTON, MICHAEL HOFFMAN AND WILLIAM HOMER
}

\begin{abstract}
For a parabolic subgroup $H$ of the general linear group $G=G l(n, \mathbf{C})$, we characterize the Kähler classes of $G / H$ and give a formula for the height of any two-dimensional cohomology class. As an application, we classify the automorphisms of the cohomology ring of $G / H$ when this ring is generated by twodimensional classes.
\end{abstract}

1. The flag manifolds. For any sequence $n_{1}, n_{2}, \ldots, n_{l}$ of positive integers with $n_{1}+n_{2}+\cdots+n_{l}=n$, let $F\left(\dot{n}_{1}, n_{2}, \ldots, n_{l}\right)$ be the space of flags $0=p_{0} \subset$ $p_{1} \subset \cdots \subset p_{l}=\mathbf{C}^{n}$ in $\mathbf{C}^{n}$ with $\operatorname{dim} p_{j}-\operatorname{dim} p_{j-1}=n_{j}$. Then $F\left(n_{1}, n_{2}, \ldots, n_{l}\right)$ can be considered as the quotient of $G l(n, \mathbf{C})$ by a parabolic subgroup, and thereby has the structure of a complex manifold of complex dimension $\sum_{p<a} n_{p} n_{q}$. In this paper we determine the heights of all elements of $H^{2}\left(F\left(n_{1}, n_{2}, \ldots, n_{l}\right) ; \mathbf{Z}\right)$

Let $s_{j}=n_{1}+\cdots+n_{j}$. Then for $1 \leq j \leq l$, we have canonical $s_{j}$-plane bundles $\xi_{j}$ over $F\left(n_{1}, n_{2}, \ldots, n_{l}\right)$. We put $x_{1}=c_{1}\left(\xi_{1}\right), x_{j}=c_{1}\left(\xi_{j}\right)-c_{1}\left(\xi_{j-1}\right)$ for $2 \leq j \leq l$. Then $H^{2}\left(F\left(n_{1}, n_{2}, \ldots, n_{l}\right) ; \mathbf{Z}\right)$ is generated by $x_{1}, x_{2}, \ldots, x_{l}$, with the single relation $x_{1}+x_{2}+\cdots+x_{1}=0$. (For a complete description of $H^{*}\left(F\left(n_{1}, n_{2}, \ldots, n_{l}\right) ; \mathbf{Z}\right)$, see [1].)

If $\iota_{1}, \iota_{2}, \ldots, \iota_{k}$ is a subsequence of $1,2, \ldots, l$ with $\iota_{k}=l$, then there is a map

$$
\iota: F\left(n_{1}, n_{2}, \ldots, n_{l}\right) \rightarrow F\left(m_{1}, m_{2}, \ldots, m_{k}\right), m_{t}=s_{\iota_{l}}-s_{\iota-1} .
$$

sending $p_{1} \subset p_{2} \subset \cdots p_{l}$ to $p_{\iota_{1}} \subset p_{\iota_{2}} \subset \cdots \subset p_{\iota_{k}}$. Let $\xi_{1}^{\prime}, \ldots, \xi_{k}^{\prime}$ be the canonical bundles over $F\left(m_{1}, m_{2}, \ldots, m_{k}\right), x_{1}^{\prime}, \ldots, x_{k}^{\prime}$ the corresponding generators of $H^{2}\left(F\left(m_{1}, m_{2}, \ldots, m_{k}\right) ; \mathbf{Z}\right)$.

Proposition 1.1. The map $\iota$ is holomorphic. Further, $\iota^{*}$ is injective and sends $x_{j}^{\prime}$ to $x_{\iota_{j-1}+1}+\cdots+x_{\iota_{j}}$.

Proof. Since both $F\left(n_{1}, n_{2}, \ldots, n_{l}\right)$ and $F\left(m_{1}, m_{2}, \ldots, m_{k}\right)$ are quotients of $G l(n, \mathbf{C})$ and the map $\iota: F\left(n_{1}, n_{2}, \ldots, n_{l}\right) \rightarrow F\left(m_{1}, m_{2}, \ldots, m_{k}\right)$ is induced by

Received by the editors November 30, 1982 and, in revised form, January 14, 1983. 1980 Mathematics Subject Classification. Primary 57T15; Secondary 53C55.

Key words and phrases. Flag manifold, height, Kähler manifold.

(C) Canadian Mathematical Society, 1983. 
the identity on $\operatorname{Gl}(n, \mathbf{C})$, we see $\iota$ is holomorphic. In fact $\iota$ is a fibration, and since its Serre spectral sequence collapses (for degree reasons), $\iota^{*}$ is an injection. Finally, we note that the bundle $\xi_{j}^{\prime}$ pulls back by $\iota$ to the bundle $\xi_{\iota j}$, and hence

$$
\begin{aligned}
\iota^{*}\left(x_{j}^{\prime}\right) & =\iota^{*} c_{1}\left(\xi_{j}^{\prime}\right)-\iota^{*} c_{1}\left(\xi_{j-1}^{\prime}\right) \\
& =c_{1}\left(\xi_{\iota_{j}}\right)-c_{1}\left(\xi_{\iota_{j-1}}\right) \\
& =x_{\iota_{j-1}+1}+x_{\iota_{\mathrm{i}-1}+2}+\cdots+x_{\iota_{j}}
\end{aligned}
$$

by naturality of Chern classes.

2. Kähler classes. Let $M$ be a complex manifold. We call a cohomology class $u \in H^{2}(M ; \mathbf{Z})$ Kähler if it projects to a Kähler class in $H^{2}(M: \mathbf{C})$. We summarize some facts about Kähler classes in the next result.

Proposition 2.1. Let $M$ be a Kähler manifold of complex dimension $d$, and suppose $u \in H^{2}(M ; \mathbf{Z})$ is Kähler.

1. The cohomology class $u$ has height $d$ in $H^{*}(M ; \mathbf{Z})$.

2. If $f: N \rightarrow M$ is a holomorphic embedding, then $f^{*} u$ is Kähler.

3. If $v \in H^{2}(M ; \mathbf{Z})$ is also Kähler, then $u+v$ is Kähler.

Proof. For (1) and (2), see [4] or [7]. For (3), note that if $M$ has Kähler classes $u$ and $v$, then $M \times M$ has Kähler class $u \otimes 1+1 \otimes v$; this goes to $u+v$ under the map induced by the diagonal embedding $\boldsymbol{M} \rightarrow \boldsymbol{M} \times \boldsymbol{M}$, so $u+v$ is Kähler by (2).

We shall obtain a formula for the height of elements of $H^{2}\left(F\left(n_{1}, n_{2}, \ldots, n_{l}\right) ; \mathbf{Z}\right)$ by showing certain elements of $H^{2}\left(F\left(n_{1}, n_{2}, \ldots, n_{l}\right)\right.$; Z) are Kähler.

The flag manifold $F\left(n_{1}, n_{2}\right)$ is the Grassmannian of $n_{1}$-planes in $\mathbf{C}^{n_{1}+n_{2}}$. The next result describes the Kähler classes in $H^{2}\left(F\left(n_{1}, n_{2}\right) ; \mathbf{Z}\right)$.

Proposition 2.2. If $a>0$, then $a x_{2}=-a x_{1} \in H^{2}\left(F\left(n_{1}, n_{2}\right) ; \mathbf{Z}\right)$ is Kähler.

Proof. The flag manifold $F(1, N)$ is just the $N$-dimensional complex projective space. Let $\xi_{1}^{\prime}$ be the canonical line bundle over $F(1, N)$, and $x_{1}^{\prime}$ the corresponding generator of $H^{2}(F(1, N) ; \mathbf{Z})$. Then $F(1, N)$ is known to have Kähler class $-x_{1}^{\prime}$ (in fact $-x_{1}^{\prime}$ projects to the class in $H^{2}(F(1, N)$; C) induced by the Fubini-Study metric on $F(1, N)$ : see [7, p. 218]). Now the Plücker embedding

$$
F\left(n_{1}, n_{2}\right) \rightarrow F(1, N), \quad N=\left(\begin{array}{c}
n_{1}+n_{2} \\
n_{1}
\end{array}\right)-1
$$

pulls back the line bundle $\xi_{1}^{\prime}$ to the line bundle $\Lambda^{n_{1}} \xi_{1}$ over $F\left(n_{1}, n_{2}\right)$ : thus, $x_{1}^{\prime}$ pulls back to $x_{1} \in H^{2}\left(F\left(n_{1}, n_{2}\right) ; \mathbf{Z}\right)$ by naturality of Chern classes. By (2) of 2.1, $-x_{1}$ is Kähler; so $-a x_{1}$ is Kähler for any $a>0$ by (3) of 2.1.

Now we can show certain classes in $H^{2}\left(F\left(n_{1}, n_{2}, \ldots, n_{l}\right) ; \mathbf{Z}\right)$ are Kähler: this 
will in fact turn out to be a complete description of Kähler classes of $F\left(n_{1}, n_{2}, \ldots, n_{l}\right)$.

TheOREM 2.3. A cohomology class $a_{1} x_{1}+\cdots+a_{l} x_{l} \in H^{2}\left(F\left(n_{1}, n_{2}, \ldots, n_{l}\right) ; \mathbf{Z}\right)$ is Kähler if $a_{1}<a_{2}<\cdots<a_{l}$.

Proof. For each $1 \leq j \leq l-1$, there is a map

$$
F\left(n_{1}, n_{2}, \ldots, n_{l}\right) \rightarrow F\left(s_{j}, n-s_{j}\right)
$$

given by picking out the $j$ th subspace in the flag. We denote the generators of $H^{2}\left(F\left(s_{j}, n-s_{j}\right) ; \mathbf{Z}\right)$ by $x_{1}^{(i)}, x_{2}^{(i)}$. (Of course $x_{1}^{(i)}=-x_{2}^{(i)}$.) Then the product of these maps,

$$
F\left(n_{1}, n_{2}, \ldots, n_{l}\right) \rightarrow \prod_{j=1}^{l-1} F\left(s_{j}, n-s_{i}\right),
$$

is a holomorphic embedding. By $1.1, x_{2}^{(j)}$ pulls back to $x_{j+1}+x_{j+2}+\cdots+x_{l}$. It follows that $a_{1} x_{1}+\cdots+a_{l} x_{l}$ is Kähler, since it is the pullback of

$$
\sum_{j=1}^{l-1}\left(a_{j+1}-a_{j}\right) x_{2}^{(j)} \in H^{2}\left(\prod_{j=1}^{l-1} F\left(s_{j}, n-s_{j}\right) ; \mathbf{Z}\right) .
$$

(We use the relation $x_{1}+x_{2}+\cdots+x_{l}=0$.)

3. Height formula and applications. We use Theorem 2.3 to prove the following formula for the height of elements of $H^{2}\left(F\left(n_{1}, n_{2}, \ldots, n_{l}\right) ; \mathbf{Z}\right)$.

THEOREM 3.1. For

$$
a_{1} x_{1}+a_{2} x_{2}+\cdots+a_{l} x_{l} \in H^{2}\left(F\left(n_{1}, n_{2}, \ldots, n_{l}\right) ; \mathbf{Z}\right),
$$

let $\left\{b_{1}<b_{2}<\cdots<b_{k}\right\}$ be the set of distinct values taken on the by the $a_{t}$, and let

$$
m_{\mathrm{j}}=\sum_{a_{\mathrm{t}}=b_{\mathrm{j}}} n_{\mathrm{t}}, \quad 1 \leq j \leq k .
$$

Then the height of $a_{1} x_{1}+\cdots+a_{l} x_{l}$ is

$$
\sum_{p<q} m_{p} m_{q}
$$

Proof. For any permutation $\sigma$ of $1,2, \ldots, l$, there is a homeomorphism

$$
F\left(n_{\sigma(1)}, n_{\sigma(2)}, \ldots, n_{\sigma(l)}\right) \rightarrow F\left(n_{1}, n_{2}, \ldots, n_{l}\right)
$$

which permutes the $x_{j}$ in cohomology. Thus, we can assume $a_{1} \leq a_{2} \leq \cdots \leq a_{l}$. If we put $\iota_{r}=$ order of $\left\{t \mid a_{t} \leq b_{r}\right\}$, then the map

$$
\iota: F\left(n_{1}, n_{2}, \ldots, n_{l}\right) \rightarrow F\left(m_{1}, m_{2}, \ldots, m_{k}\right)
$$

of 1.1 has the property

$$
\iota^{*}\left(b_{1} x_{1}^{\prime}+\cdots+b_{k} x_{k}^{\prime}\right)=a_{1} x_{1}+\cdots+a_{l} x_{l}
$$


Now $b_{1} x_{1}^{\prime}+\cdots+b_{k} x_{k}^{\prime}$ is Kähler by 2.3 , hence of height

$$
\operatorname{dim}_{\mathbf{C}} F\left(m_{1}, m_{2}, \ldots, m_{k}\right)=\sum_{\mathrm{p}<q} m_{\mathrm{p}} m_{\mathrm{q}}
$$

in $H^{*}\left(F\left(m_{1}, m_{2}, \ldots, m_{k}\right) ; \mathbf{Z}\right)$ : since $\iota^{*}$ is an injection, $a_{1} x_{1}+\cdots+a_{l} x_{l}$ must have the same height in $H^{*}\left(F\left(n_{1}, n_{2}, \ldots, n_{l}\right) ; \mathbf{Z}\right)$.

COROllary 3.2. Any Kähler class in $H^{2}\left(F\left(n_{1}, n_{2}, \ldots, n_{l}\right) ; \mathbf{Z}\right)$ must be of the form given in Theorem 2.3.

Proof. Any Kähler class

$$
u=a_{1} x_{1}+\cdots+a_{l} x_{l} \in H^{2}\left(F\left(n_{1}, n_{2}, \ldots, n_{l}\right) ; \mathbf{Z}\right)
$$

must have maximal height in $H^{*}\left(F\left(n_{1}, n_{2}, \ldots, n_{l}\right)\right.$; $\left.\mathbf{Z}\right)$, so by the previous result the coefficients $a_{j}$ must all be distinct. Suppose now that the coefficients are not in ascending order: let $a_{j}>a_{j+1}$. Choose a strictly increasing sequence $b_{1}<b_{2}<\cdots<b_{l}$ with $b_{j}=a_{j+1}$ and $b_{j+1}=a_{j}$. Then

$$
v=b_{1} x_{1}+\cdots+b_{l} x_{l} \in H^{2}\left(F\left(n_{1}, n_{2}, \ldots, n_{l}\right) ; \mathbf{Z}\right)
$$

is Kähler by 2.3. If $u$ is Kähler, then $u+v$ is Kähler by 2.1: but this is impossible, since the coefficients of $x_{j}$ and $x_{j+1}$ in $u+v$ are the same.

Let $F\left(1^{n-m}, m\right)$ denote $F\left(n_{1}, n_{2}, \ldots, n_{l}\right)$ with $n_{1}=n_{2}=\cdots=n_{l-1}=1$ and $n_{l}=m$. Then 3.1 gives us the following result.

\section{Corollary 3.3. Let}

$$
a_{1} x_{\sigma(1)}+\cdots+a_{t} x_{\sigma(t)} \in H^{2}\left(F\left(1^{n-m}, m\right) ; \mathbf{Z}\right), t \leq n-m .
$$

where $\sigma$ is a permutation of $1,2, \ldots, n$ (if $m=1$ ) or of $1,2, \ldots, n-m$ (if $m \geq 2)$ and $a_{1}, \ldots, a_{t} \neq 0$. Then

$$
t(n-t) \leq h e i g h t\left(\sum_{j=1}^{t} a_{j} x_{\sigma(j)}\right) \leq t(n-t)+\left(\begin{array}{l}
t \\
2
\end{array}\right) .
$$

with the lower bound attained if and only if all the $a_{\mathrm{j}}$ are equal, and the upper bound attained if and only if all the $a_{\mathrm{j}}$ are distinct.

Proof. Let $b_{1}, b_{2}, \ldots, b_{k}$ be as in 3.1: one of the $b_{j}$ is zero (since $t \leq n-m$ ), say $b_{r}$. Then

$$
m_{1}+m_{2}+\cdots+m_{r-1}+m_{r+1}+\cdots+m_{k}=t
$$

and $m_{r}=n-t$. Applying 3.1,

$$
\text { height } \begin{aligned}
\left(\sum_{j=1}^{t} a_{j} x_{\sigma(j)}\right) & =\sum_{p<q} m_{p} m_{q}=m_{r} \sum_{p \neq r} m_{p}+\sum_{\substack{p<q \\
p, q \neq r}} m_{p} m_{q} \\
& =(n-t) t+\sum_{\substack{p<q \\
p, q \neq r}} m_{p} m_{q} .
\end{aligned}
$$


Since

$$
0 \leq \sum_{\substack{p<q \\
p, q \neq r}} m_{p} m_{q} \leq\left(\begin{array}{l}
t \\
2
\end{array}\right)
$$

with the minimum attained precisely when there are no terms in the sum (i.e., all the $a_{i}$ are equal) and the maximum attained precisely when $m_{p}=1$ for $p \neq r$, the conclusion follows.

From the preceding result it follows that any $u \in H^{2}\left(F\left(1^{n} m, m\right) ; \mathbf{Z}\right)$ with $u^{n}=0$ is of the form $a x_{j}$. This fact is proved in [5] for the case $m \geq n-m$, and in [6] and [2] for the case $m=1$. It is used in [5] to classify automorphisms of $H^{*}\left(F\left(1^{n-m}, m\right) ; \mathbf{Z}\right)$ for $m \geq n-m$. Since 3.3 removes the restriction $m \geq n-m$, the argument of [5] gives immediately the following.

Corollary 3.4. Any automorphism of $H^{*}\left(F\left(1^{n-m}, m\right): Z\right)$ has the form

$$
x_{i} \rightarrow \varepsilon x_{q(i)}, \quad 1 \leq j \leq n-m .
$$

where $\varepsilon= \pm 1$ and $\sigma$ is a permutation of $1,2, \ldots, n$ (if $m=1$ ) or of $1,2, \ldots$, $n-m($ if $m \geq 2)$.

REMARK. The same result holds for rational coefficients, provided " $\varepsilon= \pm 1$ " is replaced by " $\varepsilon \neq 0 "$ ". It then follows that the manifolds $F\left(1^{m-n}, m\right)$ are all generically rigid, by the main result of [3].

\section{REFERENCES}

1. A. Borel, Sur la cohomologie des espaces fibrés principaux et des espaces homogènes de groupes de Lie compacts, Ann. of Math. 57 (1953), 115-207.

2. J. Ewing and A. Liulevicius, Homotopy rigidity of linear actions on homogeneous spaces, J. of Pure and Applied Alg. 18 (1980), 259-267.

3. H. Glover and G. Mislin, On the genus of generalized flag manifolds, Enseign. Math. XXVII (1981), 211-219.

4. P. Griffiths and J. Harris, Principles of Algebraic Geometry, Wiley, New York, 1978.

5. A. Liulevicius, Homotopy rigidity of linear actions: characters tell all, Bull. AMS 84 (1978), $213-221$.

6. J. D. Monk, Geometry of flag manifolds, Proc. London Math. Soc. (3) 9 (1959) 253-286.

7. R. O. Wells, Differential Analysis on Complex Manifolds. Springer-Verlag, New York, 1980.

MEMORIAL UNIVERSITY OF NFLD

ST. JOHN'S, NEWFOUNDLAND,

CANADA, A1B3X7. 